\title{
Correction: Associations Between Affective States and Sexual and Health Status Among Men Who Have Sex With Men in China: Exploratory Study Using Social Media Data
}

Zhi-Wei Zheng $^{1 *}$, MS; Qing-Ling Yang ${ }^{1 *}$, MS; Zhong-Qi Liu ${ }^{1}$, MS; Jia-Ling Qiu ${ }^{1}$, MS; Jing Gu ${ }^{1,2,3}$, PhD; Yuan-Tao $\mathrm{Hao}^{1,2,3}, \mathrm{PhD}$; Chao Song ${ }^{4}$, PhD; Zhong-Wei Jia ${ }^{5}$, PhD; Chun Hao ${ }^{1,2,3}, \mathrm{PhD}$

${ }^{1}$ Department of Medical Statistics, School of Public Health, Sun Yat-sen University, Guangzhou, China

${ }^{2}$ Health Information Research Center, Guangdong Key Laboratory of Medicine, School of Public Health, Sun Yat-sen University, Guangzhou, China

${ }^{3}$ Sun Yat-sen Global Health Institute, Institute of State Governance, Sun Yat-sen University, Guangzhou, China

${ }^{4}$ School of Computer Science and Engineering, University of Electronic Science and Technology of China, Chengdu, China

${ }^{5}$ National Institute on Drug Dependence, Peking University, Beijing, China

* these authors contributed equally

\section{Corresponding Author:}

Chun Hao, $\mathrm{PhD}$

Department of Medical Statistics

School of Public Health

Sun Yat-sen University

No 74, Zhongshan 2nd Road

Guangzhou, 510080

China

Phone: 8613922750299

Email: haochun@mail.sysu.edu.cn

\section{Related Article:}

Correction of: https://www.jmir.org/2020/1/e13201/

(J Med Internet Res 2020;22(3):e18135) doi: 10.2196/18135

In "Associations Between Affective States and Sexual and Health Status Among Men Who Have Sex With Men in China: Exploratory Study Using Social Media Data" (J Med Internet Res 2020;22(1):e13201), there was an error which was not identified during the proofing stage.

The original published Acknowledgments section was incorrectly listed as:

We are grateful to the participants of this study. This project was funded by the National Natural Science Foundation of China (grant number \#71974212, \#71774178, and \#81803334), Science and Technology Program of Guangzhou, China (grant number \#201607010332), and A Major Infectious Disease Prevention and Control of the National Science and Technology Major Project (grant number \#2018ZX10715004).
The correct Acknowledgments section is:

We are grateful to the participants of this study. This project was funded by the National Natural Science Foundation of China (grant number \#91546203, \#71974212, \#71774178, and \#81803334), Science and Technology Program of Guangzhou, China (grant number \#201607010332) and A Major Infectious Disease Prevention and Control of the National Science and Technology Major Project (grant number \#2018ZX10715004).

The correction will appear in the online version of the paper on the JMIR website on March 3, 2020, together with the publication of this correction notice. Because this was made after submission to PubMed, PubMed Central, and other full-text repositories, the corrected article has also been resubmitted to those repositories. 
This is a non-peer-reviewed article. Submitted 05.02.20; accepted 17.02.20; published 03.03.20.

Please cite as:

Zheng ZW, Yang QL, Liu ZQ, Qiu JL, Gu J, Hao YT, Song C, Jia ZW, Hao C

Correction: Associations Between Affective States and Sexual and Health Status Among Men Who Have Sex With Men in China:

Exploratory Study Using Social Media Data

J Med Internet Res 2020;22(3):e18135

URL: https://www.jmir.org/2020/3/e18135

doi: $10.2196 / 18135$

PMID: $\underline{32130135}$

(CZhi-Wei Zheng, Qing-Ling Yang, Zhong-Qi Liu, Jia-Ling Qiu, Jing Gu, Yuan-Tao Hao, Chao Song, Zhong-Wei Jia, Chun Hao. Originally published in the Journal of Medical Internet Research (http://www.jmir.org), 03.03.2020. This is an open-access article distributed under the terms of the Creative Commons Attribution License (https://creativecommons.org/licenses/by/4.0/), which permits unrestricted use, distribution, and reproduction in any medium, provided the original work, first published in the Journal of Medical Internet Research, is properly cited. The complete bibliographic information, a link to the original publication on http://www.jmir.org/, as well as this copyright and license information must be included. 